\title{
USO RECREATIVO DE BENZODIACEPINAS EN LA POBLACIÓN JOVEN.
}

\section{RECREATIONAL USE OF BENZODIAZEPINES IN THE YOUNG POPULATION}

Flavio Andrés Correa Alfaro ${ }^{1}$

María Noelia García Hernández ${ }^{2}$

1.- Enfermero. Graduado Escuela de Enfermería Ntra. Sra. de Candelaria. Servicio Canario de la Salud. Universidad de la Laguna.

2.- Enfermera. PhD. Especialista en Enfermería Pediátrica. Máster en Ciencias de la Enfermería. Máster en Bioética. Profesora Escuela Universitaria de Enfermería Ntra. Sra. de Candelaria. Servicio Canario de la Salud. Universidad de la Laguna.

Recibido: 19 de diciembre de 2018

Aceptado: 05 de febrero de 2019 


\section{Resumen:}

Introducción: el consumo de psicotrópicos y, concretamente, de benzodiacepinas, está sufriendo un notable crecimiento en Europa en los últimos años, lo que conlleva un mayor riesgo de consumo, abuso, dependencia y un empleo no médico, sobre todo en las poblaciones más jóvenes. Objetivos: revisar la bibliografía disponible sobre el uso no médico y/o recreativo de benzodiacepinas en la población joven. Material y método: se llevó a cabo una revisión bibliográfica de la literatura publicada en las bases de datos PubMed, Biblioteca Virtual de Salud, Biblioteca Cochrane Plus y Science Direct durante el periodo de un mes. Se analizó la calidad de los artículos seleccionados con instrumentos específicos según el tipo de estudio. Resultados: se localizaron un total de 980 artículos. Finalmente 10 artículos fueron incluidos por su relevancia: 2 revisiones sistemáticas, 2 estudios de cohortes y 6 epidemiológicos transversales. Todos artículos seleccionados mostraron calidad y evidencia aceptable. Los principales temas que aglutinan los resultados son motivos para el consumo sin prescripción, fuentes de adquisición y co-ingesta con otras drogas Discusión: la juventud se ha convertido en una etapa idónea para experimentar con distintas drogas, aumentando los casos de intoxicaciones y las visitas a los servicios de urgencias. La excesiva prescripción de benzodiacepinas facilita su disponibilidad y accesibilidad por parte de este grupo de población.

Palabras clave: benzodiacepinas, psicotrópicos, drogas de abuso, consumo recreativo, jóvenes.

\section{Abstract:}

Introduction: the consumption of psychotropic drugs and, specifically, of benzodiazepines, is experiencing a significant growth in Europe in recent years, which entails a greater risk of abuse consumption, dependence and non-medical use, especially in younger populations. Objectives: to review the available literature about non-medical and/or recreational use of the benzodiazepines in the young 
population. Material and method: a bibliographic review of the literature published in the databases of PubMed, Virtual Health Library, Cochrane Library Plus and ScienceDirect during the period of one month was carried out. The quality of the selected articles was analyzed with specific instruments according to the type of study. Results: a total of 980 articles were located. Finally, 10 articles were included due to their relevance: 2 systematic reviews, 2 cohort studies and 6 cross-sectional epidemiological studies. All selected articles showed quality and acceptable evidence. The main issues that agglutinate the results are reasons for consumption without prescription, sources of acquisition and co-intake with other drugs Discussion: youth has become an ideal stage to experiment with different drugs, increasing cases of poisoning and visits to the emergency services. The excessive prescription of benzodiazepines facilitates its availability and accessibility by this population group.

Keywords: benzodiazepines, psychotropic drugs, drugs abuse, recreational use, youths.

\section{Introducción}

El "nonmedical use", uso no médico en español, consiste según Fenton M. et al. (2010) (1), en usar un fármaco sin prescripción, consumirlo en mayores dosis a las prescritas, realizar ingestas más frecuentes, hacer un uso prolongado sin una justificación correspondiente o usarla con otros fines distintos a los indicados, como por ejemplo, para un uso lúdico (2). El "consumo recreativo" es aquel en el que se emplean drogas legales como tabaco, alcohol y/o psicofármacos, como ilegales, tales como cannabis y cocaína, entre otras, con el objetivo de obtener estímulos satisfactorios, disminuir efectos de otras drogas e incluso para favorecer el alcance de metas sociales o personales (2). Este tipo de consumo generalmente está asociado a tiempo y espacios de ocio vinculados principalmente a los fines de semana. 
Dentro de los denominados psicofármacos, se encuentran las benzodiacepinas (BZD). Son un grupo farmacológico conocido ampliamente desde su descubrimiento en los años 60 por su prescripción como tratamiento en el manejo del insomnio y ansiedad. Estos fármacos, en función de su composición, puede presentar, efectos ansiolítico, miorelajante, hipnótico, anticonvulsivante o inducción de amnesia anterógrada (3,4), considerándose por ello sustancias depresoras del Sistema Nervioso Central (SNC) (4). A pesar de su efecto adictivo y sus reacciones adversas a largo plazo, las BZD continúan siendo fármacos de elección para el manejo de la ansiedad e insomnio por cortos periodos de tiempo (5). El abuso de estas sustancias puede llegar a provocar un alto nivel de tolerancia y su uso prologado genera dependencia física y/o psicológica, riesgo de sufrir síntomas de abstinencia como insomnio, problemas gástricos, temblores, agitación, miedo, espasmos musculares entre otros (6). El riesgo de dependencia se asocia con su consumo prolongado, en general mayor a 3 meses, dosis elevadas o hacer uso de aquellas BDZ que posean una vida media corta con gran poder ansiolítico. El hecho de presentar tolerancia o síntomas de abstinencia al suspender el consumo, provoca que aumente su uso. Además, aquellas BDZ que generan mayores efectos satisfactorios tienen más riesgo de ser usados de forma reiterada (5). En la última década, debido a la acentuación de su popularidad, al aumento de su prescripción y al amplio abanico de efectos, ha hecho que se incluya dentro del grupo de fármacos considerados de uso habitual no médico. Este consumo nace fuera de las consultas y suelen tener fines que pueden coincidir o no con su indicación real (4).

A nivel mundial, el uso de BZD ha ido en aumento, siendo Europa uno de sus mayores consumidores. Pérez MJ et al. (2016) refieren en su artículo que existen evidencias de que alrededor de un $10 \%$ de la población de países desarrollados son consumidores de psicofármacos (5). A nivel nacional, se confirma que el aumento de consumo de ansiolíticos e hipnóticos, incluidas las BZD, ha tenido un incremento del $57.5 \%$ desde los inicios del siglo XXI (7). La Encuesta Estatal sobre Uso de Drogas en Estudiantes de Enseñanzas Secundarias (ETUDES) ${ }^{(8)}$, reafirma que el consumo de hipnosedantes se ha elevado, tanto con receta como sin ella, pasando de $8.8 \%$ y $6.8 \%$ 
en el año 2000 a 16.1\% y 7.8\% en el 2014 respectivamente. La Encuesta Estatal sobre Alcohol y otras Drogas en España (EDADES) indica que 775.762 personas de 15 a 64 años llevaron a cabo un consumo problemático de hipnosedantes durante el año $2015^{(9)}$.

Tanto jóvenes como adultos tienen posibilidades de realizar un uso incorrecto de estas sustancias. Sin embargo, la población de jóvenes adolescentes se encuentra en una etapa del desarrollo psicoemocional donde la experimentación de nuevas emociones puede conllevar acciones y asunción de riesgos para la salud en etapas tempranas de la vida (10). Se plantea la necesidad de revisar la literatura disponible con el fin de conocer las implicaciones de un consumo no médico de benzodiacepinas en jóvenes, valorando aspectos sobre motivaciones de consumo, fuentes de adquisición o consumo vinculado con otras drogas.

\section{Material y método}

Este estudio se llevó a cabo en el ámbito de la asignatura anual de Practicum del cuarto año de formación para la obtención del la titulación de Grado de Enfermería por la Universidad de La Laguna (ULL) durante el período de rotación en el ámbito de Atención Primaria (AP) de aproximadamente 7 semanas de duración. En este caso el período de prácticas clínicas abarcó desde el 11 de septiembre de 2017 hasta el 26 de octubre de 2017. El marco referencial cronológico para la elaboración del contenido de la revisión se sitúa en el mes de octubre de 2017.

La formulación de la pregunta en formato PICOT (11) para la realización de la revisión bibliográfica se muestra en la Tabla 1. Los términos DeCS utilizados fueron benzodiacepinas, psicotrópicos, drogas de abuso, consumo recreativo, jóvenes. Estos términos y sus correspondientes MeSH se presentan en la Tabla 2. Para la localización de la información se consultaron durante el período de un mes las bases de datos Biblioteca Virtual de Salud (BVS), Biblioteca Cochrane Plus, PubMed y 
ScienceDirect. Además, se localizaron de forma referencial artículos y otros documentos esenciales para el desarrollo de la revisión. En la Tabla 3 se presentan las bases de datos consultadas, la estrategia de búsqueda y sus resultados, artículos encontrados y válidos, según la fecha de consulta, los límites establecidos, las cadenas de búsqueda y los descriptores utilizados.

Como criterios de inclusión se establecieron que las investigaciones hubiesen sido publicadas en español y/o inglés en los 5 últimos años, que se centraran en un consumo recreativo, de abuso o no médico de fármacos, incluyendo a las BZD, así como a sedantes, relajantes o ansiolíticos y que la población de estudio fueran jóvenes, tanto estudiantes como no estudiantes. Los criterios de exclusión considerados fueron estudios que evaluaran poblaciones con patologías psiquiátricas, drogadicción establecida, internamiento en instituciones penitenciarias o población adulta.

El primer filtro para la selección de los artículos se realizó a través de la lectura del título y resumen y el segundo tras la lectura integra del texto de los manuscritos. La valoración de la calidad de los artículos finalmente incluidos se realizó con instrumentos de lectura crítica según el tipo de estudio. Para analizar los requisitos mínimos de evidencia de inclusión de los artículos se empleó la parrilla de lectura crítica del CASPe ${ }^{(12)}$, para las revisiones sistemáticas y estudios de cohorte, teniendo en cuenta tres aspectos generales: ¿¿Son válidos sus resultados?, ¿cuáles son los resultados?, ¿son aplicables en tu medio?”. Otra herramienta empleada para la evaluación de la calidad de los estudios epidemiológicos descriptivos fue la desarrollada por Berra S et al. (2008) (13). Este instrumento consta de 27 ítems para evaluar: pregunta u objetivo de investigación, participantes, comparabilidad entre los grupos estudiados, definición y medición de las variables principales, análisis y confusión, resultados, conclusiones, validez externa y aplicabilidad de los resultados, y conflicto de interés. Los ítems se agrupan en las siguientes dimensiones de la evaluación de la evidencia científica: 

a) pregunta u objetivo de investigación (1 ítem);
b) participantes (5 ítems);
c) comparabilidad entre los grupos estudiados (4 ítems);
d) definición y medición de las variables principales (4 ítems);
e) análisis estadístico y confusión (4 ítems);
f) resultados (4 ítems);
g) conclusiones, validez externa y aplicabilidad de los resultados (4 ítems), y
h) conflicto de intereses (1 ítem).

La calidad del estudio evaluado se puede considerar alta si la mayoría de los enunciados se responden como «muy bien»o «bien»; media si la validez interna es calificada como «regular», o la mayoría de los enunciados se responden como «bien» o «regular», y baja si la validez interna es calificada como «mal», o la mayoría de los enunciados se responden como «regular»o «mal».

El análisis cualitativo de los resultados de la revisión bibliográfica se estructurará para su presentación en áreas temáticas que incluirán motivaciones del uso, fuentes de adquisición y co-ingesta con otras drogas.

Tabla 1. Formulación pregunta investigación formato $\operatorname{PICOT}^{(11)}$.

\begin{tabular}{|l|l|l|}
\hline $\mathbf{P}$ & Paciente o problema & Uso recreativo de benzodiacepinas en jóvenes \\
\hline $\mathbf{I}$ & Intervención & Consumo sin prescripción de benzodiacepinas \\
\hline $\mathbf{C}$ & Comparación & No procede \\
\hline $\mathbf{O}$ & Resultado esperado & $\begin{array}{l}\text { Motivos de consumo sin prescripción } \\
\text { Fuentes de adquisición } \\
\text { Co-ingesta con otras drogas }\end{array}$ \\
\hline $\mathbf{T}$ & Tiempo & 1 mes (Septiembre 2017) \\
\hline
\end{tabular}




\section{Resultados}

En la Figura 1 se muestra el algoritmo del proceso de selección de estudios incluidos en esta revisión. De los 980 artículos iniciales, tras la identificación de duplicados y revisión de título y resumen, se excluyeron 627 estudios. Un total de 16 manuscritos fueron revisados en su totalidad, y 6 de ellos fueron desestimados por no cumplir con los criterios de la búsqueda o de calidad.

Finamente se incluyeron 10 artículos, 2 son revisiones sistemáticas, 2 estudios de cohortes y 6 estudios transversales. En PubMed se localizaron 6 artículos, cuatro estudios transversales y dos de cohorte. En la BVS y en ScienceDirect se localizaron una revisión sistemática y un estudio transversal en cada caso.

Se obtiene mayor número de artículos en inglés. Solo se incorporan dos artículos en español. En la Tabla 4 se presenta información relevante de los artículos incluidos en la revisión bibliográfica, identificando el tipo de estudio, los principales resultados, la puntuación obtenida en el análisis de la calidad y los niveles de evidencia.

Tabla 2. Palabras clave, términos DeCS y MeSH utilizados en las bases de datos para la localización de referencias bibliográficas.

\begin{tabular}{|c|c|c|c|}
\hline Palabra natural & DeCS español & DeCS inglés & MeSH \\
\hline Benzodiacepinas & Benzodiacepinas & Benzodiazepines & Benzodiazepines \\
Psicotrópicos & Psicotrópicos & Psychotropic drugs & Psychotropic drugs \\
Hipnóticos & Hipnóticos & Hypnotics & Hypnotics \\
& & & \\
\hline
\end{tabular}




\begin{tabular}{|l|l|l|l|}
\hline \multicolumn{1}{|c|}{ Ansiolíticos } & \multicolumn{1}{|c|}{ Ansiolíticos } & \multicolumn{1}{|c|}{ Depressants } & \multicolumn{1}{c|}{ Depressants } \\
\hline Drogas de abuso & Drogas de abuso & Drugs abuse & $\begin{array}{l}\text { Street drugs } \\
\text { Non prescription drugs }\end{array}$ \\
\hline Automedicación & Automedicación & Self medication & Self medication \\
\hline Jóvenes & $\begin{array}{l}\text { Adulto joven } \\
\text { Adolescente }\end{array}$ & $\begin{array}{l}\text { Young adult } \\
\text { Adolescent }\end{array}$ & $\begin{array}{l}\text { Young adult } \\
\text { Adolescent }\end{array}$ \\
\hline
\end{tabular}

Tabla 3. Información sobre la búsqueda bibliográfica según la base de datos consultada.

\begin{tabular}{|c|c|c|c|c|c|}
\hline Base de datos & Fecha & Descriptores y boléanos & $\begin{array}{l}\text { Artículos } \\
\text { encontrados }\end{array}$ & $\begin{array}{l}\text { Artículos } \\
\text { validos }\end{array}$ & Límites \\
\hline \multirow[b]{2}{*}{ BVS } & $19 / 09 / 17$ & $\begin{array}{l}\text { Benzodiacepinas OR } \\
\text { Hipnóticos AND Abuso }\end{array}$ & 111 & 2 & \multirow{2}{*}{$\begin{array}{l}\text { Desde } 2012 . \\
\text { Español. Texto } \\
\text { completo. } \\
\text { Acceso gratuito. }\end{array}$} \\
\hline & $20 / 09 / 17$ & $\begin{array}{c}\text { Benzodiacepinas OR } \\
\text { Psicotrópicos AND } \\
\text { Abuso }\end{array}$ & 157 & 1 & \\
\hline $\begin{array}{l}\text { Biblioteca } \\
\text { Cochrane Plus }\end{array}$ & $19 / 09 / 17$ & $\begin{array}{l}\text { (Benzodiacepinas) OR } \\
\text { (Hipnóticos) OR } \\
\text { (Psicotrópicos) OR } \\
\text { (Ansiolíticos) AND } \\
\text { Atención primaria }\end{array}$ & 22 & 1 & $\begin{array}{l}\text { Desde } 2012 . \\
\text { Español o inglés. } \\
\text { Texto completo. } \\
\text { Acceso gratuito. }\end{array}$ \\
\hline \multirow[t]{2}{*}{ PubMed } & $19 / 09 / 17$ & $\begin{array}{c}\text { Recreational drugs AND } \\
\text { Benzodiazepines OR } \\
\text { Hypnotics OR } \\
\text { Psychotropic drugs AND } \\
\text { Drugs abuse AND } \\
\text { Youths }\end{array}$ & 195 & 6 & \multirow[t]{2}{*}{$\begin{array}{l}\text { Last } 5 \text { years. } \\
\text { Free full text } \\
\text { available. }\end{array}$} \\
\hline & $20 / 09 / 17$ & $\begin{array}{l}\text { (Benzodiazepines OR } \\
\text { Hypnotics) AND Drugs } \\
\text { abuse AND Young adult }\end{array}$ & 97 & 3 & \\
\hline ScienceDirect & $20 / 09 / 17$ & $\begin{array}{c}\text { Nonmedical use AND } \\
\text { Prescription drugs AND } \\
\text { Depressants }\end{array}$ & 61 & 3 & $\begin{array}{l}\text { Últimos } 5 \text { años. } \\
\text { Texto completo. } \\
\text { Inglés o español. }\end{array}$ \\
\hline
\end{tabular}


Tabla 4. Información de los artículos incluidos en la revisión bibliográfica: autores, años de publicación principales aportaciones, nivel de evidencia con criterios de calidad (CASPe ${ }^{(12)}$ y Barre et al. ${ }^{(13)}$ ) y niveles de evidencia (SING) ${ }^{(26)}$.

\begin{tabular}{|c|c|c|c|c|}
\hline$\underset{\text { ref }}{\operatorname{Autores}(\mathbf{a n ̃ o})}$ & TE* & Principales resultados & $\mathbf{C C}^{* * *}$ & $\mathrm{NE}^{* * * *}$ \\
\hline $\begin{array}{l}\text { McCabe S, } \\
\text { Cranford J } \\
(2012)^{14}\end{array}$ & ET & $\begin{array}{l}\text { Motivaciones para consumo no médico: "sensation seeking" (30\%), "get high" } \\
(23.5 \%) \text {. } \\
\text { Motivos de automedicación: relax }(22.1 \%) \text {, dormir }(16.6 \%) \text {, mejorar estado } \\
\text { ánimo }(8.2 \%) \text {. }\end{array}$ & $\begin{array}{l}\text { Berra et al: } \\
\text { ALTA }\end{array}$ & 3 \\
\hline \multirow{3}{*}{$\begin{array}{l}\text { Bennett } \mathrm{T} \text { et al } \\
(2017)^{15}\end{array}$} & \multirow{3}{*}{ RS } & $\begin{array}{l}\text { Motivaciones: automedicación (97\%) y socialización y ambiente fiesta } \\
(48.5 \%) \text {. Fuentes de adquisición: amigos de forma gratuita }(60.5 \%) \text {, familiar de } \\
\text { forma gratuita }(28.9 \%) \text {, propia prescripción }(13.2 \%) \text {. Co-ingesta: cannabis } \\
(75 \%) \text {, alcohol }(65 \%) \text {, éxtasis }(20 \%) \text {, LSD }(20 \%) \text {, cocaína }(10 \%) \text {. }\end{array}$ & \multirow{3}{*}{$\begin{array}{l}\text { CASPe: } \\
9 / 10\end{array}$} & \multirow{3}{*}{$2++$} \\
\hline & & $\begin{array}{l}\text { Motivos para el consumo: ansiedad (32\%), "get high" (27\%), dormir (20\%), } \\
\text { otros }(21 \%) \text {. }\end{array}$ & & \\
\hline & & $\begin{array}{l}\text { Motivos consumo no médico: ansiedad }(81.3 \%) \text {, dormir }(46,6 \%) \text {, "get high" } \\
(28.4 \%) \text {, disminuir efectos de otras drogas }(16.3 \%) \text {, "sensation seeking" } \\
(9.2 \%) \text {, porque es más segura que otras drogas ilegales }(9.1 \%) \text {. No se } \\
\text { observaron diferencias entre sexos. }\end{array}$ & & \\
\hline $\begin{array}{l}\text { Schepis T et al } \\
(2016)^{16}\end{array}$ & ET & $\begin{array}{l}\text { Las motivaciones para consumo se diferenciaron según si se presentaba o no } \\
\text { una co-ingesta con otras drogas. } \\
\text { No co-ingesta: experimentación }(32 \%) \text {, "get high" }(26.9 \%) \text {, relax }(64 \%) \text {, } \\
\text { dormir }(43.7 \%) \text {. } \\
\text { Sí co-ingesta: experimentación }(55.8 \%) \text { o "get high" }(60.8 \%) \text {. } \\
\text { Consumo combinado: mayor probabilidad uso recreativo. }\end{array}$ & $\begin{array}{l}\text { Berra et al: } \\
\text { ALTA }\end{array}$ & 3 \\
\hline $\begin{array}{l}\text { Austic E et al } \\
(2015)^{17}\end{array}$ & $\mathrm{EC}$ & $\begin{array}{l}\text { Motivos consumo no médico: automedicación y el "sensation seeking”. Es más } \\
\text { frecuente en mujeres. }\end{array}$ & $\begin{array}{l}\text { CASPe: } \\
8 / 11\end{array}$ & $2+$ \\
\hline $\begin{array}{l}\text { Boyd C et al } \\
(2015)^{18}\end{array}$ & $\mathrm{EC}$ & $\begin{array}{l}\text { Motivos principales consumo no médico: automedicación, seguido del } \\
\text { "sensation seeking". A mayor tiempo de prescripción del fármaco, mayor } \\
\text { probabilidad de que el individuo haga un uso recreativo del mismo. }\end{array}$ & $\begin{array}{l}\text { CASPe: } \\
7 / 11\end{array}$ & $2+$ \\
\hline $\begin{array}{l}\text { Roncero C et al } \\
(2015)^{19}\end{array}$ & ES & $\begin{array}{l}\text { Motivos para uso no médico: estrés }(90 \%) \text {, consumo experimental }(10 \%) \text { con } \\
\text { fines no terapéuticos. Es más consumido entre mujeres que hombres }(2: 1) \text {. }\end{array}$ & $\begin{array}{l}\text { CASPe: } \\
8 / 10\end{array}$ & $2++$ \\
\hline $\begin{array}{l}\text { McCaber S, } \\
\text { West B, Teter } \\
\text { C, Boyd C } \\
(2014)^{20}\end{array}$ & ET & $\begin{array}{l}\text { Se muestra que } 1 \% \text { de los estudiantes lo obtuvieron de traficantes de drogas. } \\
\text { Las probabilidades de uso no médico de ansiolíticos era un } 34 \% \text { más baja para } \\
\text { mujeres que para los hombres. La historia de un uso médico prolongado tiene } \\
\text { altas asociaciones significativas con un mayor uso no médico. }\end{array}$ & $\begin{array}{l}\text { Berra et al: } \\
\text { ALTA }\end{array}$ & 3 \\
\hline $\begin{array}{ll}\text { McCabe } & \text { S } \\
\text { West } & \text { B } \\
(2014)^{21} & \end{array}$ & ET & $\begin{array}{l}\text { Fuentes de adquisición: principalmente amigos o familiares gratis }(71 \%) \text {, } \\
\text { comprado a amigos, familiares o traficantes de drogas }(63.8 \%) \text {. } \\
\text { Co-ingesta con otras drogas: tabaco }(16.9 \%) \text {, alcohol }(16.2 \%) \text {, otros fármacos } \\
\text { prescritos }(12 \%) \text {, cannabis }(7 \%) \text {, otras drogas ilegales }(6.9 \%) \text {. }\end{array}$ & $\begin{array}{l}\text { Berra et al: } \\
\text { MEDIA }\end{array}$ & 3 \\
\hline $\begin{array}{l}\text { Opaleye E et al } \\
(2014)^{22}\end{array}$ & ET & $\begin{array}{l}\text { Co-ingesta: alcohol }(84 \%) \text {, tabaco }(33 \%) \text {, otros fármacos }(25 \%) \text {, drogas } \\
\text { ilegales (18\%). Es más frecuente en mujeres y adolescentes con mayor poder } \\
\text { adquisitivo. La edad de mayor consumo no médico es el intervalo de 16-18 } \\
\text { años. }\end{array}$ & $\begin{array}{l}\text { Berra et al: } \\
\text { ALTA }\end{array}$ & 3 \\
\hline
\end{tabular}




\begin{tabular}{|c|c|c|c|c|}
\hline $\begin{array}{l}\text { Lucena V et al } \\
(2013)^{23}\end{array}$ & ET & $\begin{array}{l}\text { El consumo con receta era más frecuente entre estudiantes universitarios. En } \\
\text { cambio sin receta era más habitual en no universitarios. El consumo } \\
\text { experimental es mayor conforme aumenta la edad. Las mujeres presentan un } \\
\text { mayor consumo de tranquilizantes que los hombres tanto sin receta como con } \\
\text { receta. Los estudiantes universitarios tienden a tener un mayor consumo } \\
\text { combinado con otras drogas que los no universitarios. }\end{array}$ & $\begin{array}{l}\text { Berra et al: } \\
\text { MEDIA }\end{array}$ & 3 \\
\hline
\end{tabular}

* Tipo de estudio. ET: estudio transversal; RS: revisión sistemática; EC: estudio de cohortes;

** CC: Criterios de calidad

$* * * N E$ : Nivel de evidencia

Respecto a las áreas temáticas propuestas, la mayoría investigaron las motivaciones que llevan a realizar un uso no médico y recreativo de psicofármacos en jóvenes, en la que se encontraban incluidas las BZD (14-19). Tres de ellos estudiaron las fuentes de adquisición de dichos fármacos $(15,20,21)$ y cinco compararon su consumo vinculado con distintas drogas (15-23).

En la mayoría de los manuscritos $(n=9)$ se tienen en cuenta fármacos que hubieran sido prescritos por un facultativo, exceptuando el artículo de Opaleye et al. (2014) (22), en el que sólo se tiene en cuenta el consumo sin receta médica. En cuanto al perfil epidemiológico encontrado se determina que con respecto a las variables consumo-sexo, es más frecuente en mujeres que en hombres (14,21-23), en cuanto a la relación consumo-nivel educativo, solo un estudio compara el consumo entre universitarios y no universitarios (23), obteniendo que el uso no médico con prescripción médica es más frecuente en universitarios.

Se presentan a continuación los resultados agrupados según área temática analizada:

Motivos para el consumo sin prescripción. Entre las causas que incitan al consumo sin prescripción de este tipo de fármacos se encontraron la automedicación como búsqueda de acción terapéutica o motivos recreativos. El uso lúdico hace referencia a la búsqueda de nuevas sensaciones o "sensation seeking" 
en terminología anglosajona y el término coloquial de "colocarse con drogas" o su homónimo anglosajón "get high". Bennett et al. (2017) (15) introduce además otras dos causas, disminuir los efectos de otras drogas y el hecho de ser más segura que las drogas ilegales. En general, el principal motivo que consideran los autores fue la experimentación (27\%) y el segundo lugar el objetivo de drogarse (23\%) (13-19), salvo cuando existe un consumo combinado con otras sustancias, donde prevalece el "get high" (16). Schepis T et al. (2016) ${ }^{(16)}$ diferencian las motivaciones del consumo de las BZD en caso de presentarse o no una co-ingesta con otras drogas, indicando que existe una prevalencia mayor de llevar a cabo un uso recreativo de estos fármacos si se consumen otras sustancias. En cambio, Boyd C et al. (2015) (18) diferencia las distintas motivaciones según el periodo de tiempo previo de prescripción, refiriendo que a mayor tiempo de prescripción, mayor probabilidad de uso recreativo.

En relación a la automedicación todos los estudios refieren que las principales motivaciones son disminuir el estrés, favorecer el sueño y la relajación. Sólo un estudio contempló su uso para mejorar el estado de ánimo (14). Sin embargo, en todos estos casos, ninguno de los individuos seguía pautas y/o indicaciones de profesionales sanitarios, prolongando de esta forma el uso incorrecto del consumo del fármaco.

Fuentes de adquisición. La forma de adquisición podría ser de forma gratuita o de pago. Primordialmente se accede a ellos por prescripción médica, pero también se describe en la bibliografía consultada la adquisición gratuita a través de amigos o familiares que lo tuvieran prescrito, ya sea de forma consciente o inconscientemente por parte del tercero, o por compra a conocidos o pequeños traficantes $(15,20,21)$, debido a su atractivo, ya que suele tener un precio inferior a otras sustancias. 
Co-ingesta con otras drogas. La co-ingesta puede relacionarse con otras sustancias depresoras que reducen el funcionamiento del SNC, sustancias estimuladoras que incrementan el funcionamiento del SNC y/o sustancias perturbadoras que modifican las percepciones y sensaciones. Salvo en el estudio de McCabe S y West B (2014), la principal opción es el consumo combinado con sustancias depresoras mayoritariamente combinados con cannabis o alcohol (21). Sólo la revisión de Bennett T et al. (2017) tiene en cuenta el uso de otras drogas ilegales menos frecuentes, como éxtasis, LSD o cocaína, donde se observa un notable empleo combinado (10-20\%) (15). Opaleye E et al. (2014) confirma que los jóvenes que consumen alguna droga ilegal presentan una probabilidad cuatro veces mayor de hacer un uso recreativo de ansiolíticos en comparación con los que no tienen una co-ingesta (22). Según los datos de Schepis T et al. (2016), los jóvenes que realizan un consumo combinado poseen una mayor probabilidad de tener un consumo recreativo moderado o muy alto de ansiolíticos en comparación de los que no llevan un consumo combinado con otras sustancias (16). El estudio de Lucena V et al. (2013) no especifica concretamente los tipos de drogas que suelen combinarse con estos fármacos, pero sí que los estudiantes universitarios tienden a tener un mayor consumo combinado de riesgo con otras drogas que los no universitarios (23).

\section{Discusión}

Los resultados obtenidos han permitido obtener información relevante para cumplir con el objetivo principal de esta revisión bibliográfica. Se ha podido incorporar información sobre el uso no médico y/o recreativo de las BZD en la población joven. Sin embargo, los resultados reflejan escasez de investigaciones predominando los estudios de tipo descriptivo de prevalencia, estudios epidemiológicos transversales (14,16, 20-23), frente a estudios analíticos (17-19) o de revisión (15). Se evidencia también poca producción de artículos cuyo objetivo de estudio se centre exclusivamente en el consumo recreativo de dichos fármacos. 
Como limitación de esta revisión bibliográfica consideramos que podrían haber estudios publicados en otros idiomas que puedan cubrir esta carencia que describimos y, que aún siendo estudios relevantes no hayan sido incluidos por el límite de idioma, español o inglés, que se ha establecido en el diseño de esta revisión bibliográfica.

En los estudios publicados la juventud es considerada por los distintos autores como una etapa perfecta para satisfacer el desarrollo de nuevas experiencias y el contacto con iguales. Este proceso suele generar situaciones de estrés que puede incrementar el empleo de distintas drogas tanto ilegales como legales $(14,16,19)$. Se sitúa el intervalo entre los 16 y 18 años como la franja de edad con mayor riesgo de consumo no médico ${ }^{20)}$.

Aunque la bibliografía describe que la población de consumidores recreativos de BZD no es muy elevada como ocurre con otro tipo de sustancias relacionadas con el consumo lúdico, las cifras de automedicación están en aumento (15). Este hecho puede estar relacionado con el actual aumento de prescripción de este grupo de fármacos lo que hace posible poder hallarlos con mayor frecuencia en los botiquines de los hogares. Boyd et al (2015) ${ }^{(18)}$ refieren en su estudio que cuanto mayor sea la duración de tiempo de prescripción del fármaco mas probabilidades de aumentar la posibilidad de consumo recreativo del mismo. También influye la prescripción en el tipo de población joven que puede acceder a través de esta vía, ya que según la bibliografía revisada son los estudiantes universitarios los que acceden con receta y tienden a ser uso combinado con otras drogas mientras los no universitarios acceden por otras vías como amistades o familiares (21-23). Esta consideración es importante a la hora de elaborar e implementar estrategias de intervención para los distintos grupos de jóvenes, sin obviar la necesidad de control de calidad en la prescripción de este grupo de fármacos. 
Es de destacar que son varios los estudios, Lucena et al (2013) (23), McCabe et al (2014)(20) y Roncero et al (2015) (23), los que describen como mayor y más frecuente el consumo de este tipo de sustancias en el sexo femenino, duplicando incluso la frecuencia de consumo respecto al sexo masculino. Esta singularidad de género resulta de interés para ser considerada en futuros estudios que permitan profundizar en las causas que pudieran estar generando estas diferencias.

Por otro lado, el reconocimiento legal de las sustancias puede facilitar su accesibilidad, y por tanto, su consumo y las consecuencias en salud derivadas de él, provocando un incremento del número de visitas a los servicios de salud y urgencias por intoxicaciones. Además, el consumo de más de una sustancia, la combinación de distintos psicótropos en el mismo acto de consumo y/o el consumo conjunto con otro tipo de droga legal como puede ser el tabaco o el alcohol, multiplica los riesgos de intoxicación. Es necesario profundizar en el análisis de las facilidades de acceso a las sustancias legalizadas y en el impacto de la potenciación de los efectos tóxicos en el consumo de varias sustancias conjuntas en la población joven, así como los factores que orientan a este grupo de población hacía favorecer o rechazar el consumo de benzodiacepinas como uso recreativo(21,22).

Es interesante considerar en este punto que la taxonomía enfermera NANDA (24) incorpora diagnósticos de enfermería con este tipo de enfoque como el diagnóstico de enfermería [00126] “Conocimientos deficientes", definido como "carencia o deficiencia de información cognitiva relacionada con un tema específico". La identificación de falta de información en este grupo de población es un pilar fundamental en la cual las enfermeras pueden contribuir en la mejora de la situación desde su campo de actuación. La Clasificación de Intervenciones de Enfermería, NIC (25), propone intervenciones específicas enfermeras para el abordaje de este diagnóstico como pueden ser la intervención [4500] "Prevención del consumo de sustancias nocivas" o [8272] "Fomentar el desarrollo: adolescente" 
contribuyendo desde la óptica de empoderamiento de la población joven, trabajando con su autorresponsabilidad en la conservación de su salud.

Todos los artículos presentes en esta revisión poseen una buena calidad metodológica. Sin embargo, algunos estudios evalúan las variables mediante cuestionarios autoadministrados $(14,16,20-23)$, por lo que sería necesario un abordaje cualitativo más profundo para conocer los factores que incentivan el consumo o, más importante aún, los factores preventivos que podrían estar ejerciendo efecto para contrarrestar el consumo de este tipo de sustancia. En este sentido, el abordaje desde este última perspectiva podría arrojar información enriquecedora sobre los aspectos que los jóvenes consideran importantes para rechazar el consumo de psicótropos de uso recreativo. El análisis cualitativo de la perspectiva de los jóvenes para evitar el consumo de sustancias nocivas en el ámbito recreativo, la implementación de intervenciones enfermeras específicas para la prevención del consumo y el análisis de su coste-efectividad de estas intervenciones se convierte en una línea futura de estudio de sumo interés.

\section{Bibliografía}

1. Fenton M, Keyes K, Martins S, Hasin D (2010): "The role of a prescription in anxiety medication use, abuse and dependence”. Am J Psychiatry, 167 (10): pp. 1247-1253.

2. Solari-Twadell PA et al. (1991): "Recreational drugs: societal and professional issues". Nurs Cli North Am, 26 (2): pp. 499- 509.

3. Domínguez V, Collares M, Ormaechea G (2016): "Uso racional de benzodiacepinas: hacia una mejor prescripción". Rev urug med interna, 3: pp. 14-24. 
4. Moratilla Díaz C (2014) "Hipnóticos-Sedantes. Una aproximación desde la gestión de placeres y riesgos", en Martínez Oró DP, Pallarés Gómez J: De riesgos y placeres. Manual para entender las drogas. Lleida: Editorial Milenio, pp. 379- 388.

5. Pérez MJ, Veas P (2014): "Uso prolongado de benzodiacepinas y estrategias para su deshabituación". Cuad Med Soc, 54 (1): pp. 8-18.

6. López Vantour A, Aroche Arzuaga A, Bestard Romero J, Ocaña Fontela N (2010): "Uso y abuso de las benzodiacepinas". MEDISAN, 14 (4): pp. 555-566.

7. Agencia Española de Medicamentos y Productos Sanitarios (2014) Utilización de medicamentos ansiolíticos e hipnóticos en España durante el periodo 2000-2012. Madrid: Ministerio de Sanidad y política social.

8. Plan Nacional Sobre Drogas (2015) Encuesta Estatal sobre Uso de Drogas en estudiantes de enseñanzas secundarias (ESTUDES) 2014/2015. Madrid: Ministerio de Sanidad, Servicios Sociales e Igualdad.

9. Plan Nacional Sobre Drogas (2016) Encuesta Estatal sobre alcohol y otras drogas (EDADES) 2015/2016. Madrid: Ministerio de Sanidad, Servicios Sociales e Igualdad.

10. Rojas Valero MJ, Rodríguez Chacón AB (2013) Abuso de drogas en adolescentes y jóvenes y vulnerabilidad familiar. Lima, Perú: Oficina de las Naciones Unidas contra la Droga y el Delito (UNODC).

11. Peñaherrera Oviedo C, Soria Viteri J (2015): "Pregunta de investigación y estrategia PICOT”. Rev. Med. FCM-UCSG, 19 (1): pp. 66-69.

12. CASPe (2005) Guías CASPe de Lectura Crítica de la Literatura Médica. Alicante: CASPe.

13. Berra S, Elorza-Ricart JM, Estrada MD, Sánchez E (2008): "Instrumento para la lectura crítica y la evaluación de estudios epidemiológicos transversales". Gaceta Sanitaria, 22: pp. 492-497. 
14. McCabe S, Cranford J (2012): "Motivational subtypes of nonmedical use of prescription Medications: results from a national study". Jorunal of Adolescent Health, 51: pp. 445-452.

15. Bennett T, Holloway K (2017): "Motives for illicit prescription drug use among university students: a systematic Review and meta-analysis". International Journal of Drug Policy, 44: pp. 12-22.

16. Schepis T, West B, Teter C, McCabe S (2016): "Prevalence and correlates of co-ingestion of prescription tranquilizers and other psychoactive substances by US high school seniors: results from a national survey". Psychol Addict Behav, 52: pp. 8-12.

17. Austic E, McCabe S, Stoddard S, Epstein Ngo Q, Boyd C (2015): "Age and cohort patterns of medical and nonmedical use of controlled medication among adolescents". J Addict Med, 9 (5): pp. 376-382.

18. Boyd C, Meier E, Espetin Q, Veliz P, McCabe S (2015): "A prospective study of adolescents' Nonmedical use of anxiolytic and sleep medication". Psychol Addict Behav, 29 (1): pp. 184-191.

19. Roncero C, Egido A, Rodríguez Cintas L, Pérez Pazos J, Collazos F, Casas M (2015): "Consumo de drogas entre los estudiantes de medicina: una revisión de la literatura 1988-2013". Actas Esp Psiquiatr, 43 (3): pp. 109-21.

20. McCabe S, West B, Teter C, Boyd C (2014): "Trends in medical use, diversión, and Nonmedical use of prescription Medications among college students from 2003 to 2013: connecting the dots". Pychol Addict Behav, 39 (7): pp. 1176-1182.

21. McCabe S, West B (2014): "Medical and nomedical use of prescription benzodiazepine anxiolytics among U.S. high school seniors". Psychol Addict Behav, 39 (5): pp. 959-964.

22. Opaleye E, Ferri CP, Locatelli DP, Amato TC, Noto AR (2014): "Nonprescribed use of tranquilizers and use of other drugs among Brazilian students". Revista Brasileira de Psiquiatría, 36: pp. 16-23. 
23. Lucena V, Ruiz Olivares R, Pino MJ, Herruzo J (2013): "Consumo de alcohol, tabaco y psicofármacos en jóvenes universitarios y no universitarios". Psicología Conductual, 21 (1): pp. 123-136.

24. NANDA International (2013). NANDA International, Diagnósticos enfermeros: Definiciones y Clasificación, 2012-2014. 2ª ed. Barcelona: Elsevier.

25. Bulechek G, Butcher H, Dochteman J, Wagner C (2013). Clasificación de Intervenciones de Enfermería (NIC). 6ª edición. Barcelona: Elsevier.

26. Harbour R, Miller J (2001): “A new system for grading recommendations in evidence bases guidelines". BMJ, 323: pp. 334-336. 